\title{
Phosphorylation of the insulin receptor by AMP-activated protein kinase (AMPK) promotes ligand-independent activation of the insulin signalling pathway in rodent muscle
}

\author{
I. Chopra • H. F. Li • H. Wang • K. A. Webster
}

Received: 10 September 2011 / Accepted: 10 November 2011 /Published online: 30 December 2011

(C) Springer-Verlag 2011

\begin{abstract}
Aims/hypothesis Muscle may experience hypoglycaemia during ischaemia or insulin infusion. During severe hypoglycaemia energy production is blocked, and an increase of AMP:ATP activates the energy sensor and putative insulinsensitiser AMP-activated protein kinase (AMPK). AMPK promotes energy conservation and survival by shutting down anabolism and activating catabolic pathways. We investigated the molecular mechanism of a unique glucose stress defence pathway involving AMPK-dependent, insulin-independent activation of the insulin signalling pathway.

Methods Cardiac or skeletal myocytes were subjected to glucose and insulin-free incubation for increasing intervals up to $20 \mathrm{~h}$. AMPK, and components of the insulin signalling pathway and their targets were quantified by western blot using phosphor-specific antibodies. Phosphomimetics were used to determine the function of IRS-1 Ser789 phosphorylation and in vitro $\left[{ }^{32} \mathrm{P}\right]$ ATP kinase assays were used to measure the phosphorylation of the purified insulin receptor by AMPK.

Results Glucose deprivation increased Akt-Thr308 and Akt-Ser473 phosphorylation by almost tenfold. Phosphorylation of glycogen synthase kinase 3 beta increased in parallel, but phosphorylation of ribosomal 70S subunit-S6 protein kinase and mammalian target of rapamycin decreased. AMPK
\end{abstract}

Electronic supplementary material The online version of this article (doi:10.1007/s00125-011-2407-y) contains peer-reviewed but unedited supplementary material, which is available to authorised users.

I. Chopra $\cdot$ H. F. Li $\cdot$ H. Wang $\cdot$ K. A. Webster $(\bowtie)$

Department of Molecular and Cellular Pharmacology,

University of Miami Miller School of Medicine,

1600 NW 10th Ave, RMSB 6038,

Miami, FL 33136, USA

e-mail: kwebster@med.miami.edu inhibitors blocked and aminoimidazole carboxamide ribonucleotide (AICAR) mimicked the effects of glucose starvation. Glucose deprivation increased the phosphorylation of IRS-1 on serine-789, but phosphomimetics revealed that this conferred negative regulation. Glucose deprivation enhanced tyrosine phosphorylation of IRS-1 and the insulin receptor, effects that were blocked by AMPK inhibition and mimicked by AICAR. In vitro kinase assays using purified proteins confirmed that the insulin receptor is a direct target of AMPK. Conclusions/interpretation AMPK phosphorylates and activates the insulin receptor, providing a direct link between AMPK and the insulin signalling pathway; this pathway promotes energy conservation and survival of muscle exposed to severe glucose deprivation.

Keywords AICAR · AMPK · Glucose transport . Hypoglycaemia · Insulin resistance $\cdot$ Insulin sensitivity Insulin signalling $\cdot$ IRS- 1 . Serine/threonine kinase $\cdot$ Tyrosine kinase

$\begin{array}{ll}\text { Abbreviations } \\ \text { ACC } & \text { Acetyl coenzyme A carboxylase } \\ \text { Ad } & \text { Adenovirus } \\ \text { AICAR } & \text { Aminoimidazole carboxamide ribonucleotide } \\ \text { AMPK } & \text { AMP-activated protein kinase } \\ \text { CAP } & \text { Cb1-associated protein } \\ \text { CBL } & \text { Casitas B-lineage lymphoma } \\ \text { dn } & \text { Dominant negative } \\ \text { dnAMPK } & \text { Dominant-negative AMPK adenovirus } \\ \text { DOG } & \text { 2-Deoxy-[ }{ }^{3} \text { H]glucose } \\ \text { GFP } & \text { Green fluorescent protein } \\ \text { GSK3 } \beta & \text { Glycogen synthase kinase } 3 \text { beta } \\ \text { IR } & \text { Insulin receptor } \\ \text { IRK } & \text { Insulin receptor kinase } \\ \text { JNK1 } & \text { c-Jun N-terminal kinase 1 }\end{array}$




$\begin{array}{ll}\text { mTOR } & \text { Mammalian target of rapamycin } \\ \text { p70S6K } & \text { Ribosomal 70S subunit-S6 protein kinase } \\ \text { PI3K } & \text { Phosphatidylinositol 3-kinase } \\ \text { PKC } & \text { Protein kinase C } \\ \text { S6K } & \text { S6 protein kinase } \\ \text { SIK2 } & \text { Salt-inducible kinase 2 } \\ \text { TC10 } & \text { Ras-like protein TC10 } \\ \text { TORC1 } & \text { Target of rapamycin complex 1 } \\ \text { TSC2 } & \text { Tuberous sclerosis complex-2 }\end{array}$

\section{Introduction}

The insulin and 5'-AMP-activated protein kinase (AMPK) signalling pathways regulate glucose and fatty acid metabolism, and numerous components of growth, differentiation and survival. Both pathways are ubiquitously expressed in eukaryotic tissues [1,2]. Activation of the insulin signalling pathway is an anabolic stimulus, while AMPK represses anabolism and activates catabolic pathways to conserve ATP. The insulin signalling pathway is activated when insulin or IGF-1 bind the insulin receptor (IR), activate the insulin receptor kinase (IRK) and autophosphorylate the IR. The activated IR binds and tyrosine phosphorylates IRS proteins, creating docking sites for $\mathrm{SH} 2$ domain-containing proteins that propagate the signal to the final effectors $[1,3]$. The major pathways docking at the activated IRSs are the phosphatidylinositol 3-kinase (PI3K) pathway, the Cb1-associated protein (CAP)-casitas B-lineage lymphoma (CBL)-ras-like protein TC10 (TC10) pathway, and the mitogen-activated protein kinase and the protein kinase $\mathrm{C}$ (PKC) pathways $[1,4,5]$. PI3K, CAP-CBL-TC10 and PKC are the major conduits for glucose (GLUT4) regulation. PI3K recruits the Akt kinase, phosphoinositide-dependent kinase-1, and Akt is activated by phosphorylation of Thr308. As described in recent reviews, Akt phosphorylates several targets that regulate cell growth and survival, as well as metabolism [6, 7]. The targets include caspase 9, the pro-apoptotic B cell leukaemia/lymphoma 2 (BCL2) family member, BCL2-associated agonist of cell death (BAD), the forkhead transcription factors, glycogen synthase kinase 3 beta $(\mathrm{GSK} 3 \beta)$ and the ribosomal $70 \mathrm{~S}$ subunit-S6 protein kinase (p70S6K) [8-10].

The activities of the IR and IRS proteins are modulated by serine/threonine (Ser/Thr) phosphorylation. Ser/Thr phosphorylation typically confers negative regulation and insulin resistance, as described by others [11-13]. This regulation mostly takes place at the level of IRS-1/2. IRSer1275, $-1309,-1293$ and -1294 are subject to autophosphorylation by IRK, and Ser117, -78, -82, -1035 and -1037 are phosphorylated by PKC [14]. IRS proteins have several $\mathrm{Ser} / \mathrm{Thr}$ sites, most of which negatively regulate signal transduction and confer insulin resistance. As described previously and in reviews, IRS proteins contain targets for Akt, S6 protein kinase (S6K), c-Jun N-terminal kinase 1 (JNK-1), PKC, GSK3 $\beta$, salt-inducible kinase 2 (SIK2) and AMPK [11-13]. IRS-1-Ser307 is a key regulatory site that, when phosphorylated by JNK-1, inhibitor of $\mathrm{KB}$ kinase beta (IKK- $\beta$ ) or PKC, inhibits signal conduction. IRS-1-Ser636 and -639 phosphorylation by S6K also blocks signal transduction, and mediates negative feedback and termination of insulin signalling [15-17]. SIK2 and AMPK phosphorylate IRS-1 at the same Ser789 site [18-22]. Whereas positive regulation by Ser789 has been suggested [20], such positive regulation has not been confirmed, and in light of more recent studies including the results described here, it seems likely that Ser789 exerts only negative regulation of IRS-1 [18, 19, 21, 22].

AMPK is an energy sensor kinase that responds to the ATP:ADP/AMP energy charge of the cell. It is activated allosterically by AMP binding and phosphorylation by liver kinase 1 [23]. As reviewed by others, AMPK is activated in muscle by physiological and pathophysiological stimuli that increase AMP levels, including contraction, hypoxia and hypoglycaemia [24, 25]. The activity of AMPK is also modulated by hormones and cytokines that affect wholebody energy balance, and by the insulin-sensitising drugs metformin and the thiazolidinediones, as described in a recent review [25]. AMPK activity is increased by glucose deprivation of muscle cells, which correlates with increased glucose uptake and glycolysis independently of insulin [2628]. AMPK also enhances fatty acid transport and oxidation, while switching off fatty acid, cholesterol, glycogen and protein synthesis pathways. The latter effects contribute to the 'insulin-sensitising' properties of AMPK [29]. AMPK blocks cell growth both indirectly by inhibiting the activity and production of biosynthetic enzymes, and directly by targeting the tuberous sclerosis complex-2 (TSC2) and target of rapamycin complex 1 (TORC1)-raptor, thereby blocking phosphorylation of p70S6K.

Several pathways have been described for AMPKenhanced insulin sensitivity and glucose transport; a major molecular component involves relief of the negative feedback loop and inhibition of S6K by phosphorylation of TSC2 [25]. Here, we present evidence for another link between AMPK and the insulin signalling pathway via direct phosphorylation and ligand-independent activation of the IR.

\section{Methods}

Reagents Antibodies directed against Akt, phosphor-AktThr308, phosphor-Akt-Ser473, AMPK, phosphor-AMPKThr172, IRS-1, phosphor-IRS-1-Ser789, GSK3 $\beta$, 
phosphor-GSK3 $\beta$-Ser9, phosphor acetyl coenzyme A carboxylase (ACC)-Ser79, anti-green fluorescent protein (GFP) and phosphor-IRS-1 (panTyr) (ELISA) were from Cell Signaling Technology (Beverly, MA, USA). PI3K, actin, IR, phosphorIR Tyr1158, Tyr1162 and Tyr1163 antibodies, and AMPK $(\alpha 1, \beta 1, \gamma 1)$ active were from Millipore (Temecula, CA, USA). Anti-Myc antibody was from Sigma (St. Louis, MO, USA). Insulin was from Sigma, and LY294002 and wortmannin were from BioSource (Camarillo, CA, USA). Aminoimidazole carboxamide ribonucleotide (AICAR), compound C and Akt inhibitor X were from EMD4 Biosciences (San Diego, CA, USA). 2-Deoxy- $\left[{ }^{3} \mathrm{H}\right]$ glucose (DOG) was from Amersham Biosciences (Piscataway, NJ, USA) and $\left[\gamma^{3}{ }^{32} \mathrm{P}\right]$ ATP was from Perkin Elmer (San Jose, CA, USA). The ATP colorimetric assay kit was from BioVision (Mountain View, CA, USA) and the QuikChange Site-Directed Mutagenesis Kit was from Stratagene (La Jolla, CA, USA). The recombinant adenoviral vector expressing dominant negative (dn)AMPK was a generous gift from M.J. Birnbaum (Institute of Obesity, Diabetes and Metabolism, University of Pennsylvania, Philadelphia, PA, USA).

Cell culture Methods for primary culture of neonatal rat cardiac myocytes have been previously described [30]. Before experiments, cells were transferred to defined serum and glucose-free DMEM, supplemented with transferrin and vitamin B12, with or without insulin as indicated. For viral infections, cultures were exposed to the indicated amount of virus for $48 \mathrm{~h}$ before exposure to experimental conditions. Cultures were exposed to AMPK-modifying drugs, including Ara-A, compound C or AICAR, $30 \mathrm{~min}$ before collection or as indicated. Intact mouse myotubes were isolated from dispersed hindlimb muscles by incubation with collagenase and dispase, and plating on collagen-coated dishes as described [31]. Preparations containing intact myofibres and myotubes were exposed to serum-free defined medium before experimental treatments. All experiments involving animals were approved by the University of Miami Institutional Animal Care and Use Committee.

Western blot, immunoprecipitation and immunostaining Detailed procedures for western blot analyses and immunostaining have been described [30]. Immunoprecipitation assays followed the same procedures as western blots, except that cleared lysates were pre-incubated with antibody overnight and complexes separated using protein-G-agarose before gel electrophoresis.

Site-directed mutagenesis Methods described by Luo et al. [32] were used to implement mutagenesis of IRS-1-Ser794. Briefly, human IRS1 cDNA was inserted into a pReceiverLv08 vector and amplified with PCR using a kit (QuikChange Site-Directed Mutagenesis; Stratagene). The primers were 5'-
CACTGCCTCTGGTCGCCTTCTCTATG-3' (Ser-Ala) or 5'CACTGAATCTGGTCGCCT TCTCTATG-3' (Ser-Glu). Mutations were verified by sequencing. Adenoviruses (Ad) encoding GFP-IRS-1 or mutants were produced using RAPAd CMV Adenoviral Expression System (Cell Biolabs, San Diego, CA, USA). Myocytes were infected with the viruses at a multiplicity of infection of 50 for $48 \mathrm{~h}$ before treatments.

Glucose uptake measurement Glucose was measured using DOG as described by Chaudary et al. [33]. Briefly, cells were washed three times with KRB buffer at $37^{\circ} \mathrm{C}$. Glucose uptake was initiated by adding $0.1 \mathrm{mmol} / 1 \mathrm{DOG}$ containing $37 \mathrm{kBq} / \mathrm{ml} \mathrm{DOG}$, and cells were incubated at $37^{\circ} \mathrm{C}$ for 5 to $15 \mathrm{~min}$. At each time point, cells were chilled on ice, washed three times with ice-cold KRB containing $25 \mathrm{mmol} / \mathrm{l}$ glucose, lysed with $\mathrm{NaOH}$, neutralised and counted by scintillation.

In vitro PI3K activity assay PI3K activity associated with IRS-1 was analysed following the procedure described elsewhere [34]. IRS-1 was immunoprecipitated from cell lysates and reactions incubated at room temperature using $2 \mu \mathrm{g} / \mu \mathrm{l}$ phosphatidylinositol and $\left[\gamma_{-}{ }^{32} \mathrm{P}\right]$ ATP $(740 \mathrm{kBq})$. The PI3K phosphorylation product was visualised by thin-layer chromatography and autoradiography.

In vitro AMPK assay Equal aliquots of IR immunoprecipitated from serum-starved HepG2 cell lysates were mixed with kinase buffer, $0.5 \mathrm{mmol} / 1$ AMP, active AMPK (3 ng/ $/ \mu \mathrm{l}), 25 \mu \mathrm{mol} / 1$ ATP and $\left[\gamma^{-32} \mathrm{P}\right]$ ATP $(3.7 \times$ $10^{5} \mathrm{~Bq}$ ), and incubated at $30^{\circ} \mathrm{C}$ for 5 to $20 \mathrm{~min}$. Reactions were stopped by boiling in SDS-loading buffer. Proteins were separated by PAGE and labelled products detected by autoradiography.

Statistical analysis Western blots were quantified using NIH Image J software (http://rsbweb.nih.gov/ij/ downloaded June 2011). Results are expressed as mean \pm SEM. Differences between means were evaluated by two-tailed Student's $t$ test.

\section{Results}

Activation of Akt in glucose- and insulin-depleted myocytes Cardiac myocytes were subjected to glucose- and insulin-free medium for progressive time periods up $24 \mathrm{~h}$ and components of the insulin signalling pathway measured at intervals. As indicated in Fig. 1, the phosphorylation of Akt on Thr308 and Ser473 peaked between 4 and $8 \mathrm{~h}$ at almost tenfold above the levels of parallel glucosecontaining cultures and remained elevated over $24 \mathrm{~h}$. GSK3 $\beta$ phosphorylation increased in parallel, whereas the phosphorylation of S6K and mammalian TORC1 Ser2448 
a

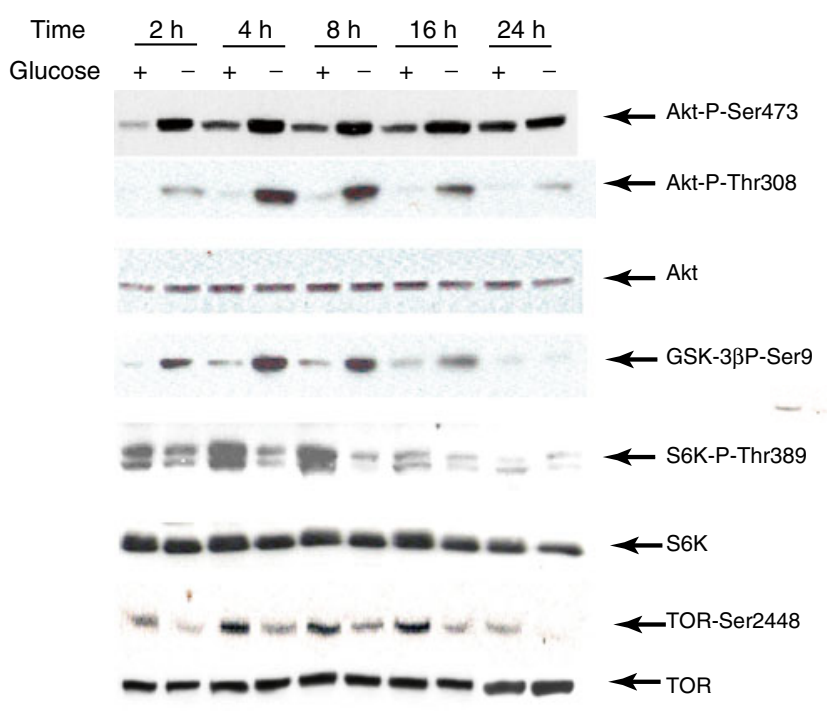

b

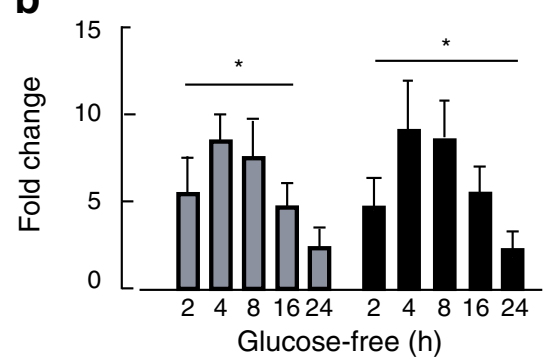

C

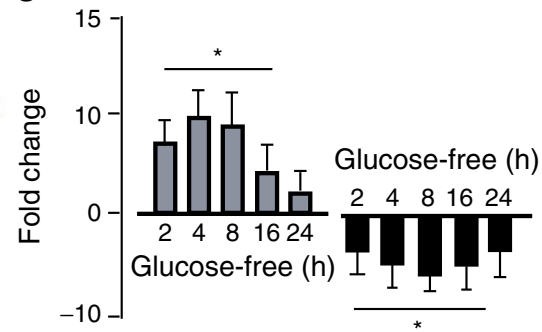

d Glucose-free $(\mathrm{h})$

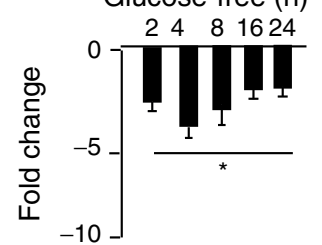

Fig. 1 Activation of the insulin signalling pathway proteins by glucose deprivation. a Cardiac myocytes were cultured in defined medium without insulin or glucose for $24 \mathrm{~h}$ and plates were removed at the indicated times for western blot analyses as described. P, phosphorylation. b Western blots of Akt phosphorylation at Ser473 (grey bars)

decreased in the glucose-free condition. GSK $3 \beta$ is a direct substrate for Akt, whereas $\mathrm{S} 6 \mathrm{~K}$ is phosphorylated by the mammalian TORC1 $[6,10]$. TORC1 is negatively regulated by $\mathrm{TSC} 2$, and $\mathrm{S} 6 \mathrm{~K}$ phosphorylation is normally increased transiently by insulin because Akt phosphorylates and inactivates TSC2 [35]. Activated S6K then contributes to the negative feedback regulation of the insulin signal by phosphorylating IRS-1-Ser636 and -639, thereby inhibiting PI3K $[15,16,36]$. Downregulation of S6K simultaneously with activation of Akt-Thr308 and -Ser473 suggests that glucose starvation mimics insulin stimulation upstream but not downstream of Akt.

Glucose starvation activates PI3K To investigate the effect of glucose starvation on signalling components upstream of Akt, we measured PI3K activity and the binding of the $\mathrm{p} 85$ subunit of PI3K to IRS-1 (Fig. 2a, b). Glucose starvation caused a significant increase of PI3K activity at 4 and $8 \mathrm{~h}$, which coincided with the peak of Akt phosphorylation and with increased binding of PI3K p85 to IRS-1. PI3K activity did not correlate with Akt phosphorylation at later time points, suggesting that additional factors contribute to the and Thr308 (black bars) were quantified by densitometry in the linear exposure range using NIH Image $J$ as described. c Quantification as above (b) of GSK3 $\beta$ (grey bars) and S6K (black bars) phosphorylation, and (d) mTOR phosphorylation at Ser2448; $n=3 ;{ }^{*} p<0.05$ by Student's $t$ test

regulation of Akt when glucose starvation is prolonged. To confirm the role of PI3K, we pretreated cardiac myocytes with the partially selective inhibitors LY294002 and wortmannin, exposed cultures to glucose deprivation for $4 \mathrm{~h}$ and quantified Akt phosphorylation as described in Fig. 1. As shown in Fig. 2c, Akt phosphorylation at Thr308 was fully blocked ( $>95 \%$ ) by both inhibitors, whereas Akt phosphorylation at Ser473 was unaffected by these inhibitors under normal glucose conditions and partially $(40 \pm 9 \%)$ inhibited by LY294002 in the glucose-deprived condition.

Glucose starvation primes insulin signalling To determine whether enhanced IRS-1/PI3K activity caused by glucose depletion augments insulin signalling, cardiac myocytes were cultured in the presence or absence of glucose as described (Fig. 1) and stimulated with insulin. As shown in Fig. 2d, e, insulin phosphorylation of Akt-Thr308 and GSK3 $\beta$ was markedly enhanced by glucose-free culture. As expected, Akt and GSK3 $\beta$ basal phosphorylation were elevated in glucose-starved cultures prior to insulin stimulation. Glucose starvation conferred about a fourfold enhancement of insulin-stimulated Akt-Thr308 and a twofold enhancement of 


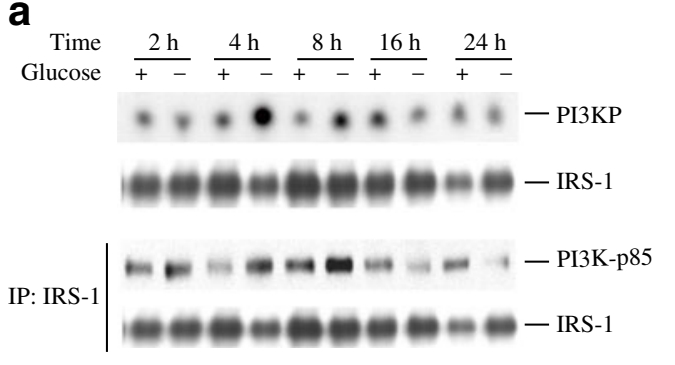

b

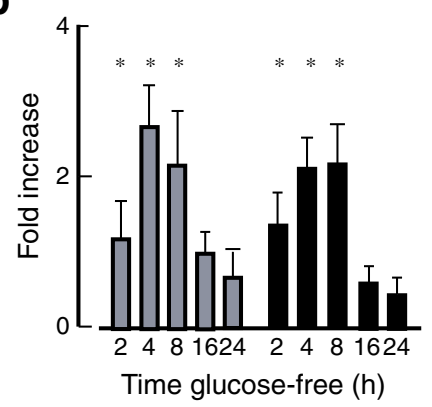

Fig. 2 Glucose starvation activates PI3K and confers enhanced insulin sensitivity. a Cardiac myocytes were cultured as described (Fig. 1). At each time point, cell lysates were immunoprecipitated (IP) with antiIRS-1 antibody and PI3K was assayed as described, or lysates were subjected to western blot analysis with antibodies against the p85 subunit of PI3K or IRS-1. PI3K-P, phosphorylated PI3K. b Quantification of PI3K-P and $\mathrm{p} 85$ in blots (a) from three separate experiments; ${ }^{*} p<0.05$. c Akt-Thr308 phosphorylation (Akt-P-T308) is sensitive to PI3K inhibitors. Cardiac myocytes were cultured as described (Fig. 1) and incubated with $(+)$ or without $(-)$ glucose (Glu) for $4 \mathrm{~h}$ in the presence and absence of LY294002 $(20 \mu \mathrm{mol} / \mathrm{l})$ or wortmannin $(\mathrm{Wm}$,

GSK $3 \beta$ at both insulin concentrations. Therefore glucose starvation primes insulin signalling and is a powerful stimulus of the pathway with or without insulin.

Glucose starvation activates AMPK, an acute effect of which is to phosphorylate TSC2 and raptor, and to block the activation of mammalian TORC1 and S6K [35, 37]. This antagonises the action of insulin on TORC1 and S6K. To begin investigating a possible role of AMPK in the regulation of Akt by glucose deprivation, we quantified the phosphorylation of its principal target, ACC, during insulin stimulation in the presence and absence of glucose. As shown in Fig. 2d, the activity of AMPK as reflected by phosphorylated ACCSer79 was markedly increased in glucose-free cultures. To determine whether treatment with AICAR also primed insulin signalling, we repeated the experiments described in Fig. 2d, but substituted AICAR treatment for glucose deprivation. As
C

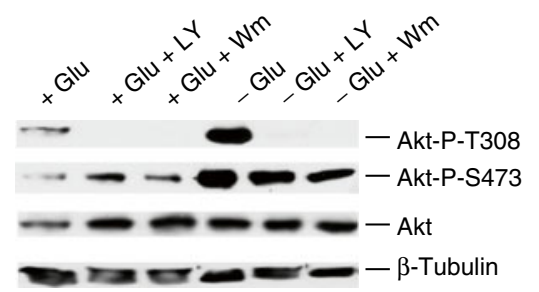

d

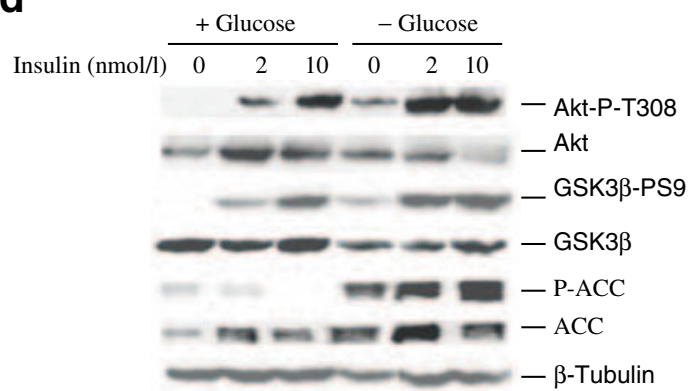

e
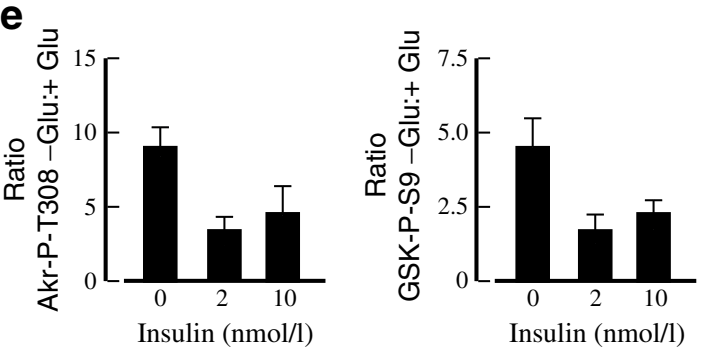

$10 \mu \mathrm{mol} / \mathrm{l})$ as indicated. Results are representative of three experiments. Akt-P-S473, Akt-Ser473 phosphorylation. d Glucose deprivation enhances insulin-induced Akt phosphorylation. Cardiac myocytes were incubated for $20 \mathrm{~h}$ with $(+)$ or without $(-)$ glucose, stimulated with $100 \mathrm{nmol} / \mathrm{l}$ insulin for the indicated time periods and equal cell lysate proteins analysed by western blot with the indicated probes. GSK3 $\beta$-P-S9, phosphorylation of GSK3 $\beta$ at Ser9; P-ACC, phosphorylated ACC. e Akt-P-T308 was quantified as the ratio of Akt-P-T308: total Akt. Glucose starvation significantly increased insulin-induced phosphorylation of Akt at 5 and $15 \mathrm{~min}, p<0.01 ; n=4$

shown in electronic supplementary material (ESM) Fig. 1, AICAR pretreatment for 30 min increased the phosphorylation of AMPK and ACC, and enhanced the phosphorylation of Akt-Thr308 in the presence and in the absence of insulin. These results are consistent with a role for AMPK in the activation of the insulin signalling pathway by glucose deprivation.

Stimulation of Akt by glucose starvation requires AMPK Figures 1 and 2 show that glucose starvation differentially regulates Akt and S6K, and activates AMPK. To determine whether AMPK is involved in the reciprocal regulation of Akt and $\mathrm{S} 6 \mathrm{~K}$ by glucose depletion, we again monitored AMPK activity through its markers phosphorylated ACC and phosphorylated AMPK, and determined the effects of AMPK inhibitors and AICAR. These results are 
shown in Fig. 3a-d. In Fig. 3a cardiac myocytes were again subjected to glucose- and insulin-free incubation for $4 \mathrm{~h}$, this time in the presence and absence of dnAMPK Ad (dnAMPK) or Ad-GFP as control. Phosphorylation of Akt on Thr308 and Ser473 was increased by glucose starvation and this was blocked by dnAMPK. Similarly ACC phosphorylation on Ser79 was increased by glucose starvation and blocked by dnAMPK. Consistent with previous reports, we found that IRS-1-Ser789 phosphorylation was markedly increased by glucose starvation, again in an AMPK-dependent manner. Expression of the cMyc tag confirmed high expression of the dnAMPK, and the effect on Akt was paralleled by phosphorylation of GSK3 $\beta$ Ser9 (Fig. 3a). To further investigate a role for AMPK in these effects, cardiac myocytes were starved of glucose for $4 \mathrm{~h}$ in the presence and absence of the AMPK inhibitor Ara-A, or treated with AICAR for 30 min with normal glucose. As shown in Fig. 3b, glucose starvation increased the phosphorylation of ACC, AMPK, Akt, GSK3 $\beta$ and IRS-1-Ser789, this effect being blocked in each case by Ara-A and mimicked by AICAR. To confirm these effects over an extended time course, myocytes were subjected to glucose starvation for up to $16 \mathrm{~h}$ as described (Fig. 1) and the phosphorylation of ACCSer79 and of three key regulatory IRS-1 sites was quantified. As shown in Fig. 3c, d, phosphorylation of ACC-Ser79 was increased by about twofold at all time points. IRS-1-Ser789 phosphorylation was increased by more than fivefold, but there was no significant change in the phosphorylation of a

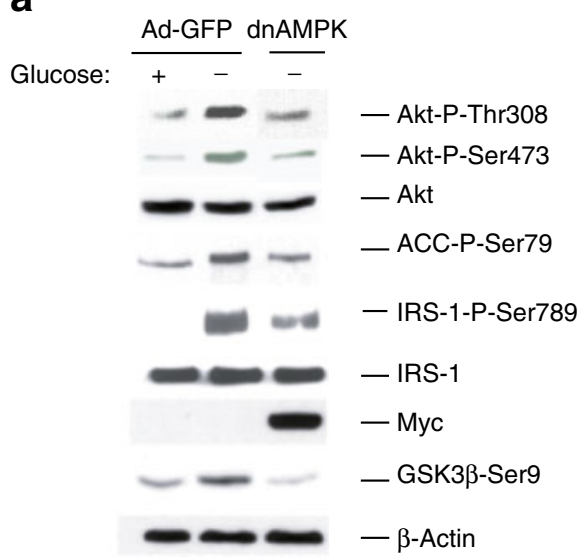

b

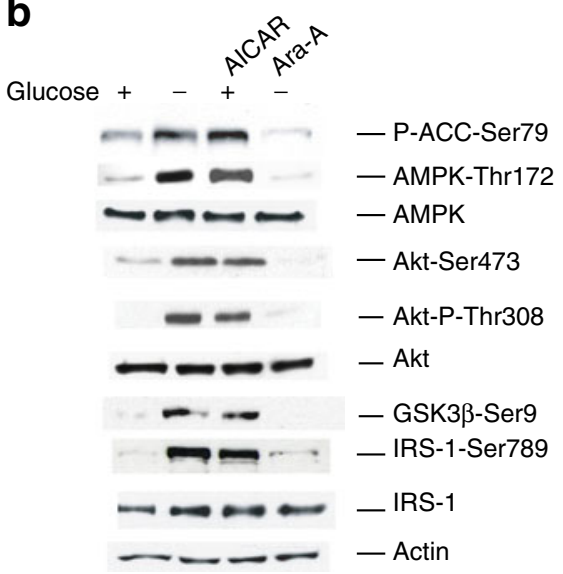

C Time $\frac{2 \mathrm{~h}}{+-} \frac{4 \mathrm{~h}}{+-} \frac{8 \mathrm{~h}}{+-} \frac{16 \mathrm{~h}}{+-}$ $-\cdots-\cdots-$ P-ACC-Ser79 $--\infty-\infty$ AMPK $--m--m---$ IRS-1-P-Ser636/639 $--\infty--\infty-\infty$ IRS-1-P-Ser307 $-m-m-m-m-$ IRS-1-P-Ser789 $-\infty-\infty-\cdots-\operatorname{IRS}-1$

d

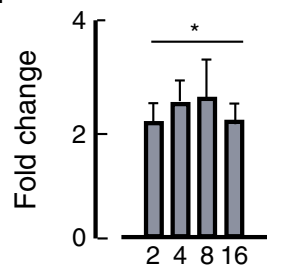

e

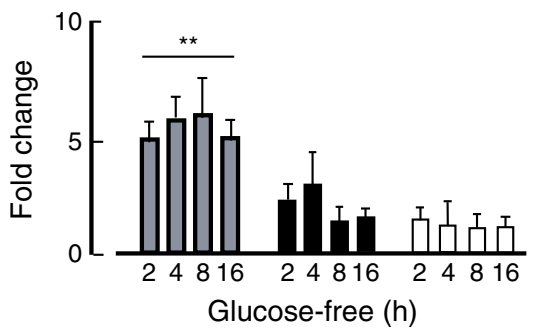

was at $5 \mathrm{mmol} / \mathrm{l}$ for $1 \mathrm{~h}$ before cell collection. Results are representative of three separate experiments. c Cardiac myocytes were cultured with or without glucose as described (Fig. 1a) and equal proteins were analysed by western blot with the indicated probes. $\mathbf{d}$ Western blots of ACC-P were quantified as described and corrected for protein loading; please note that total ACC protein levels were not significantly affected by glucose starvation (not shown). e Quantification as above (d) of IRS-1 phosphorylation at Ser789 (grey bars), Ser636 and Ser639 (black bars), and Ser307 (white bars). d, e $n=4 ;{ }^{*} p<0.05$ and ${ }^{* *} p<0.01$; NS, IRS-1 phosphorylation at Ser636 and Ser639 and Ser307 
IRS-1 Ser636, -639 or -307 . The absence of an effect on IRS$1-$ Ser636 and -639 is consistent with the suppression of S6K (Fig. 1). In control experiments we found that insulin treatment caused the rapid phosphorylation of IRS-1-Ser307, -636 and -639 as well as S6K, but not of IRS-1-Ser789 (ESM Fig. 2). Taken together, these results show that Akt activation by glucose starvation requires AMPK activation.

Negative regulation of Akt by IRS-1-Ser789 To determine whether the increased phosphorylation of IRS-1-Ser789 contributes to the activation of Akt, mutations were introduced into human GFP-tagged IRS1 cDNA to replace Ser794 (human IRS-1-Ser794=rat IRS-1-Ser789) with alanine (IRS-1-Ser794-A) or glutamine (IRS-1-Ser794-E) to respectively block or mimic phosphorylation. IRS-1 wildtype and mutant cDNAs were cloned into adenoviral vectors and used to infect cardiac myocytes prior to exposure to glucose starvation as described. The results of these experiments are shown in Fig. 4. Glucose-free culture increased the phosphorylation of Akt on Thr308 and Ser473 as expected, this being markedly enhanced in each case by overabundance of wild-type IRS-1, indicating that endogenous IRS-1 is limiting in the activation of Akt. Infection of cultures with IRS-1-Ser794-A increased Akt and GSK3 $\beta$ phosphorylation to a level comparable with wild-type IRS1, whereas infection with IRS-1-Ser794-E decreased Akt phosphorylation relative to wild-type or uninfected cells. Quantification of these results is shown in Fig. 4b. The phosphorylation of Akt-Thr308 was increased eightfold in glucose-starved myocytes infected with wild-type IRS-1 or the Ser-Ala mutation, whereas overexpression of the SerGlu mutant caused $>50 \%$ loss of Akt-Thr308 phosphorylation. In data not shown, we found the same trends when AICAR treatments replaced glucose starvation. Phosphorylation of Akt on Ser473 displayed similar trends, but was less dependent on IRS-1, a finding that is consistent with the alternative pathway of activation [38]. Figure 4c shows that infection of cardiac myocytes with the different adenoviral vectors generated equivalent IRS-1-GFP levels. The results support previous reports $[18,19,21,22]$ that the IRS-1Ser789 (h794) site confers negative regulation and cannot

Fig. 4 Phosphomimetic analyses of IRS-1-Ser789/794 function. Cardiac myocytes were infected with IRS-1 wild-type (wt), or with IRS-1 mutations with glutamine (IRS-1-S794-E) or alanine (IRS-1-S794-A), each with a GFP tag. Infection was for $48 \mathrm{~h}$ as described above (Methods), followed by $4 \mathrm{~h}$ incubation in glucose-free media. a Equal cell lysate proteins were analysed by western blot for Akt phosphorylation (P), GSK3 $\beta$-P and IRS-1. The upper band in the IRS-1 lanes indicates levels of infected IRS-1-GFP. S, Ser; T, Thr. b Quantification of Akt-P abundance by Ad infection, relative to control (no Ad, i.e. wt); $n=4 ;{ }^{\dagger} p<0.004$ and ${ }^{* *} p<0.0001$. c Cardiac myocytes were infected with each Ad as described (Methods) and visualised for GFP fluorescence, immunostained GFP antibody and DAPI. Results showing equivalent GFP abundance with each Ad are representative of three separate infections. A, alanine; E, glutamine; S, Ser account for enhanced Akt activation by glucose starvation or AMPK.

Glucose depletion increases tyrosine phosphorylation of IRS-1 Because glucose starvation caused increased binding of IRS-1 to PI3K, we determined whether this was accompanied

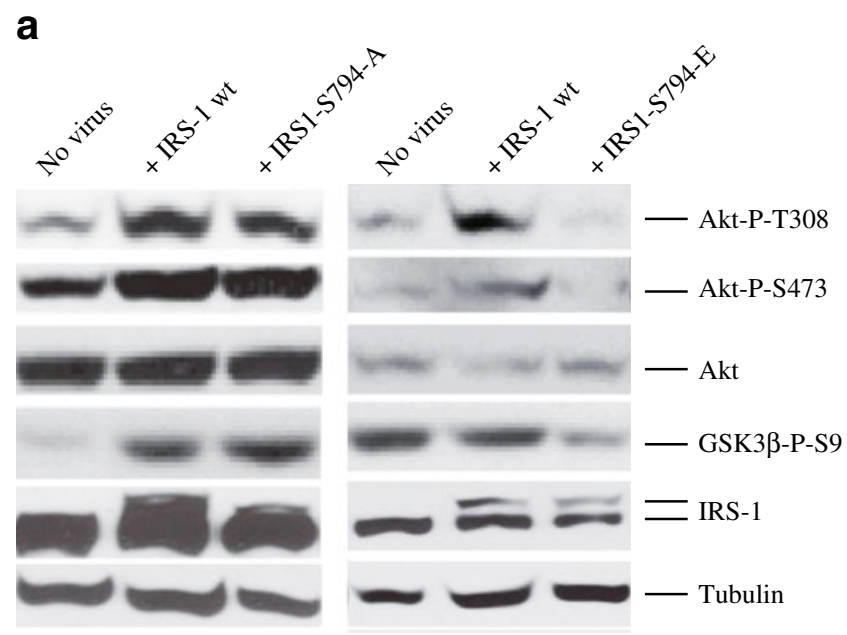

b

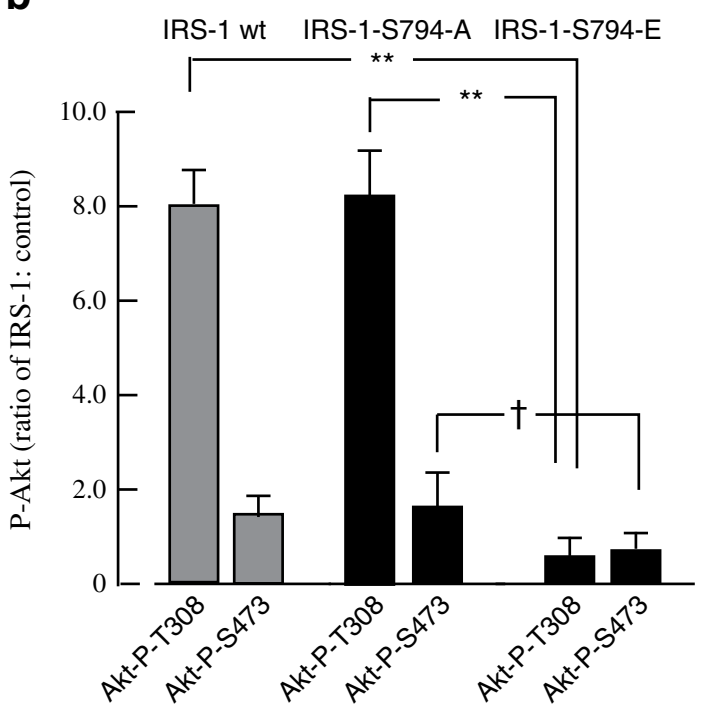

C

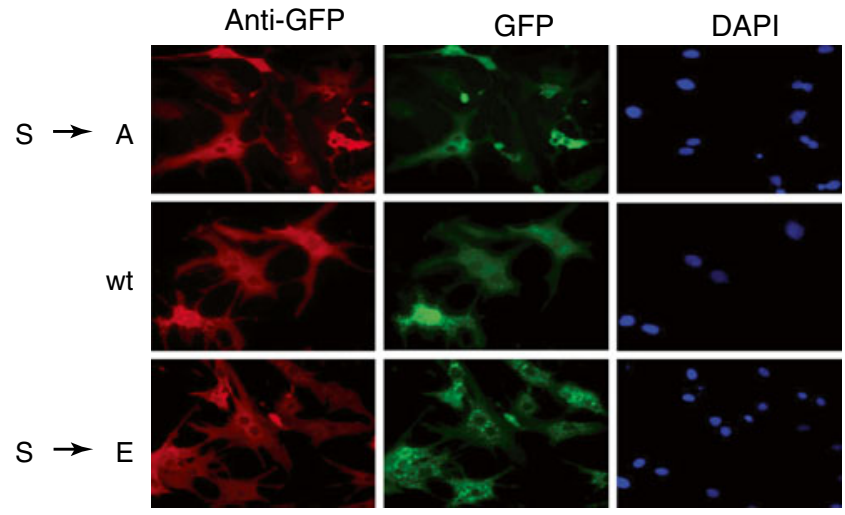


by changes in IRS-1 tyrosine phosphorylation. Total IRS-1 phosphotyrosine was quantified by ELISA before and after treatments with insulin or glucose-free incubation as described (Methods). As shown in Fig. 5a, tyrosine phosphorylation of IRS-1 was significantly increased by insulin stimulation or glucose starvation. IRS-1 residues Tyr612 and Tyr632 are within the PI3K binding domain of IRS-1 and essential for this binding [39]. Therefore we also examined the effects of glucose starvation and insulin on the phosphorylation of these IRS-1 residues. Figure $5 \mathrm{~b}$ shows that glucose starvation or treatment with AICAR increased the phosphorylation of both tyrosine residues by amounts that were close to that caused by insulin treatment. Figure $5 \mathrm{~b}$ also shows that selective inhibition of AMPK with compound C eliminated the increased phosphorylation of Tyr632 simultaneously with parallel inhibition of Akt and ACC phosphorylation. Quantification of these results (Fig. 5c) confirmed that glucose starvation mediated a significant AMPK-dependent increase in the phosphorylation of IRS1 at Tyr632.

Glucose depletion increases tyrosine phosphorylation of IR Activated IRK is the only known pathway for tyrosine phosphorylation of IRS-1. We therefore asked whether increased tyrosine phosphorylation of IR paralleled that of IRS-1. Previous studies have shown that IR activation loop tyrosine residues 1162 and 1163 are essential for activation of the IRK by insulin binding [40]. As shown in Fig. 6a, b, phosphorylation of IR at Tyr1162 increased significantly during glucose starvation. This was paralleled by increased
Akt and GSK $3 \beta$ phosphorylation, and blocked by dnAMPK. The lower mobility band representing AMPKGFP (Fig. 6a) confirms overabundance of dnAMPK. We found that compound $\mathrm{C}$ also inhibited the enhanced phosphorylation of IR at Tyr1162 by glucose starvation (not shown). Therefore glucose starvation increases tyrosine phosphorylation of key residues of the IR and IRS-1 in an AMPK-dependent manner. As expected, IR Tyr1162 phosphorylation was also increased by AICAR treatment (ESM Fig. 3). To determine whether these results extended to skeletal myocytes and intact myotubes, we measured Akt, IRS-1-PY632 and/or IR-PY1162 in C2C12 skeletal myocytes, and in freshly isolated primary mouse myofilaments and myotubes (see Methods) after exposure to glucose-free incubation. As shown in Fig. 6c, d, Akt-Thr308, IRS-1Y632 and IR-Y1162 phosphorylation were each increased by glucose starvation or insulin treatment of $\mathrm{C} 2 \mathrm{C} 12$ myocytes (Fig. 6c) and intact myotubes (Fig. 6d), suggesting that similar pathways operate in skeletal muscle. For myotubes, we also demonstrated that GSK3 $\beta$ was phosphorylated in parallel and all effects were mimicked by AICAR (Fig. 6d).

Phosphorylation of IR by AMPK Figures 5 and 6 show that glucose starvation mediates the AMPK-dependent increase in tyrosine phosphorylation of IRS-1 and IR. Therefore we hypothesised that AMPK directly phosphorylates the IR. To test for this, we mixed purified $\alpha$-AMPK and immunoprecipitated IR $\beta$-subunit in an in vitro kinase reaction. As shown in Fig. 7, the IR $\beta$-subunit has a molecular size of a

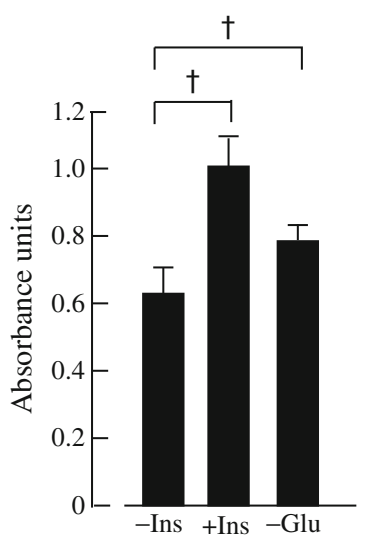

b

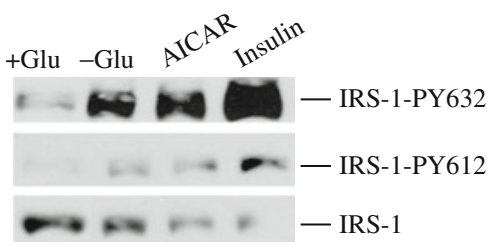

C

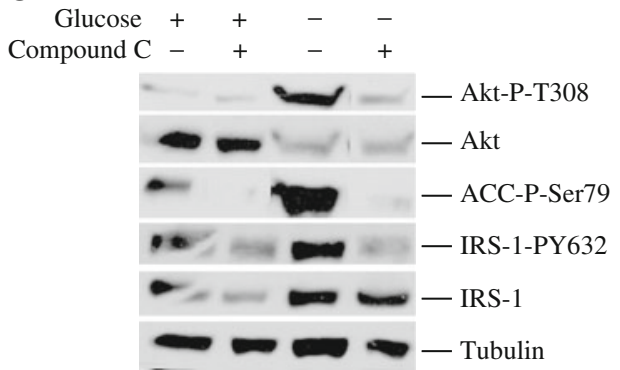

d

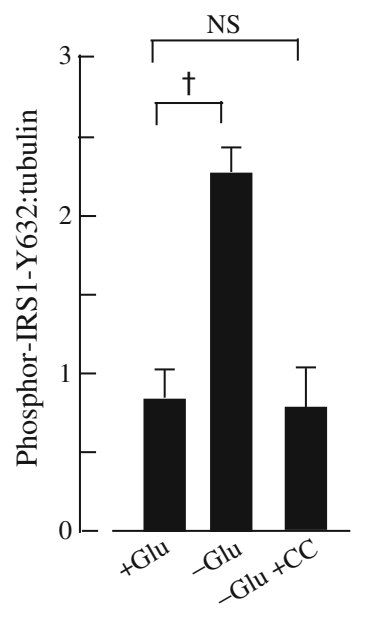

Fig. 5 Glucose starvation mediates AMPK-dependent IRS-1 tyrosine phosphorylation. a Cardiac myocytes were cultured as described (Fig. 1a) and incubated with or without glucose for $4 \mathrm{~h}$. Total IRS-1 phosphor-tyrosine was measured on equal protein cell lysates using an ELISA kit. For the positive control (with insulin, +Ins), cultures were stimulated with $100 \mathrm{nmol} / 1$ insulin for $7 \mathrm{~min} ; n=4$; ${ }^{\dagger} p<0.003$. b Cardiac myocytes were cultured as above (a), incubated with $(+)$ or without (-) $5 \mathrm{mmol} / 1$ glucose for $4 \mathrm{~h}$, and treated with $0.5 \mu \mathrm{mol} / \mathrm{l}$ AICAR for $30 \mathrm{~min}$ or with $100 \mathrm{nmol} / \mathrm{l}$ insulin for $7 \mathrm{~min}$. Equal lysate proteins were analysed by western blot using the indicated probes. $\mathbf{c}$ Cultures were additionally treated with or without compound C (20 $\mu \mathrm{mol} / \mathrm{l})$ for $30 \mathrm{~min}$ before collection. d Phosphor-IRS-1 Y632 was quantified as the ratio of phosphorylated IRS-1-Y632/total IRS-1; $n=4 ;{ }^{\dagger} p<0.0002$. P, phosphorylation; Y, Tyr 

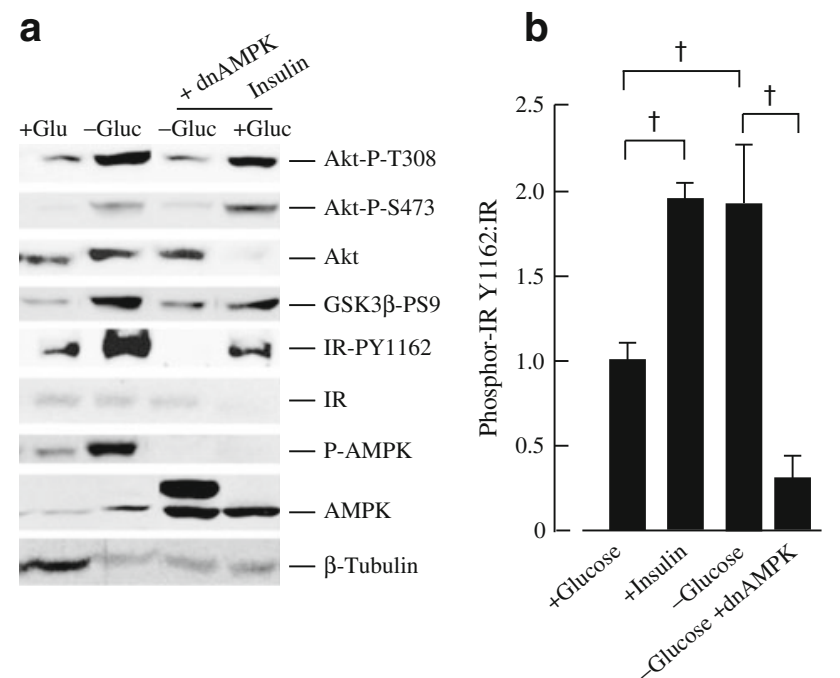
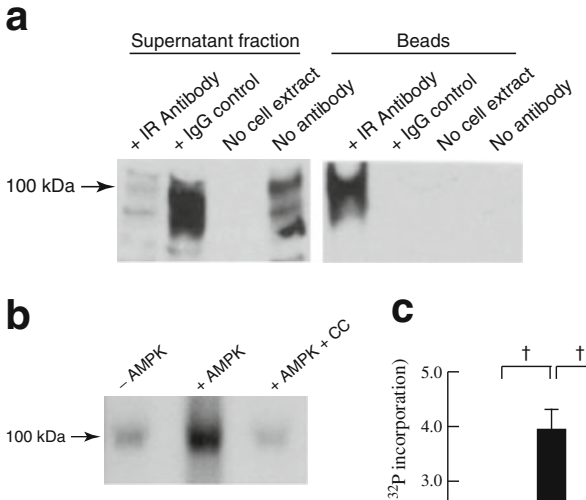

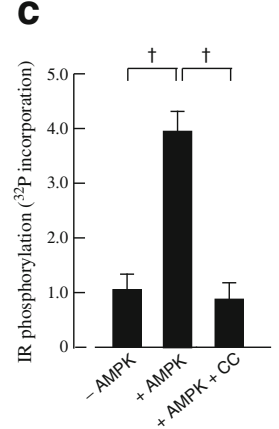

C

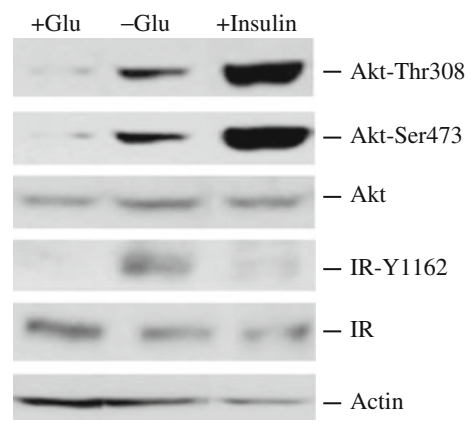

d

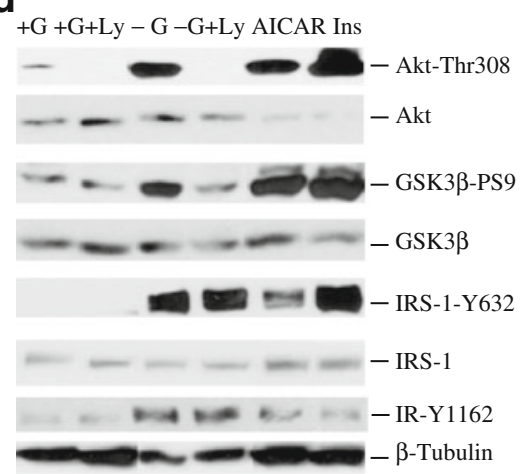

Fig. 6 Glucose starvation mediates IRS-1 and IR tyrosine phosphorylation in cardiac and skeletal myocytes, and isolated skeletal muscle fibres. a Cardiac myocytes were infected or not with dnAMPK as described (Methods), followed by culture as described (Fig. 1a) and incubation with or without glucose for $4 \mathrm{~h}$. Insulin treatment was $100 \mathrm{nmol} / 1$ for $7 \mathrm{~min}$. Equal cell lysate proteins were analysed by western blot. P, phosphorylation; S, Ser; T, Thr; Y, Tyr. b PhosphorIR Y1162 was quantified as the ratio of phosphorylated Y1162:total IR; $n=4 ;{ }^{\dagger} p<0.005$. c C2C12 skeletal myocytes were serum-starved overnight and incubated for $4 \mathrm{~h}$ without insulin and with or without $5 \mathrm{mmol} / \mathrm{l}$ glucose. For insulin-treated lanes, cultures were treated with $100 \mathrm{nmol} / 1$ insulin for $10 \mathrm{~min}$. Equal lysate proteins were analysed by western blot as indicated. $\mathbf{d}$ Skeletal myotubes were isolated from mouse hindlimbs as described (Methods) and incubated in defined medium with or without glucose (G), LY294002 (Ly, $20 \mu \mathrm{mol} / \mathrm{l})$, AICAR $(0.5 \mu \mathrm{mol} / 1$ for $30 \mathrm{~min})$ or insulin (100 nmol/1 for $10 \mathrm{~min}$ ). Equal lysate proteins were analysed by western blot. Results are representative of at least three experiments
Fig. 7 Phosphorylation of the IR by AMPK. a HepG2 cells cultured without serum or insulin for $24 \mathrm{~h}$ were collected and cell lysates used for immunoprecipitation of the IR as described (Methods). Proteins were fractionated by SDS-PAGE. Lanes show proteins remaining in the supernatant fraction or attached to the protein-A beads after immunoprecipitation. b IR immunoprecipitates containing equal proteins were incubated with or without AMPK and $\left[{ }^{32} \mathrm{P}\right] \mathrm{ATP}$ as described (Methods) in the presence and absence of $20 \mu \mathrm{mol} / 1$ compound $\mathrm{C}$ (CC). Proteins were separated by SDS-PAGE, followed by detection of phosphorylated IR by autoradiography. c Autoradiograms (b) were quantified by densitometry using NIH Image $\mathrm{J} ; n=3 ;{ }^{\dagger} p<0.0001$

about $100 \mathrm{kDa}$ (Fig. 7a). In the absence of AMPK, we observed a small incorporation of $\left[{ }^{32} \mathrm{P}\right]$ ATP into the $100 \mathrm{kDa}$ putative IR band that may represent endogenous IR autophosphorylation or contaminating kinases. There was a marked increase in the phosphorylation of IR when AMPK was included in the reaction, and this was eliminated by inclusion of the AMPK inhibitor compound C (Fig. 7b), confirming AMPK-selective phosphorylation of IR in vitro. Quantification confirmed significant AMPK-dependent, compound-C-sensitive incorporation of $\left[{ }^{32} \mathrm{P}\right] \mathrm{ATP}$ into the IR (Fig. 7c).

Akt-dependent preservation of intracellular ATP The energy-conserving roles of AMPK are well documented; however, Akt has also been shown to preserve intracellular ATP [41]. To see whether Akt activation during glucose starvation conserved energy, we measured ATP in glucosestarved cardiac myocytes in the presence and absence of the PI3K inhibitor LY294002 and the Akt inhibitor Akt-X. As shown in Fig. 8, intracellular ATP levels declined significantly at 4 and $6 \mathrm{~h}$ of glucose depletion, but recovered significantly at $8 \mathrm{~h}$ in a PI3K- and Akt-dependent manner.

AMPK- and PI3K-dependent glucose uptake We predicted that simultaneous activation of AMPK and Akt by 
a

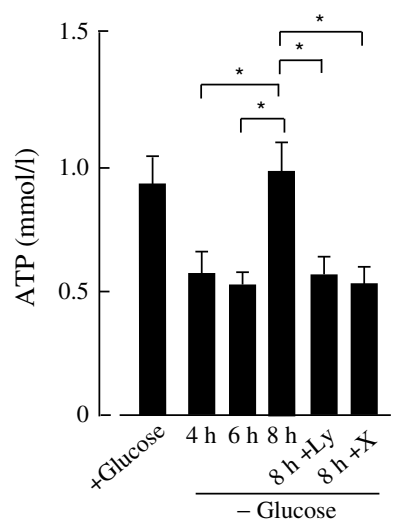

b

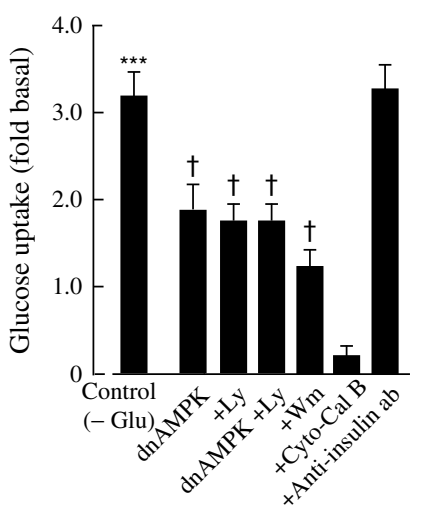

C

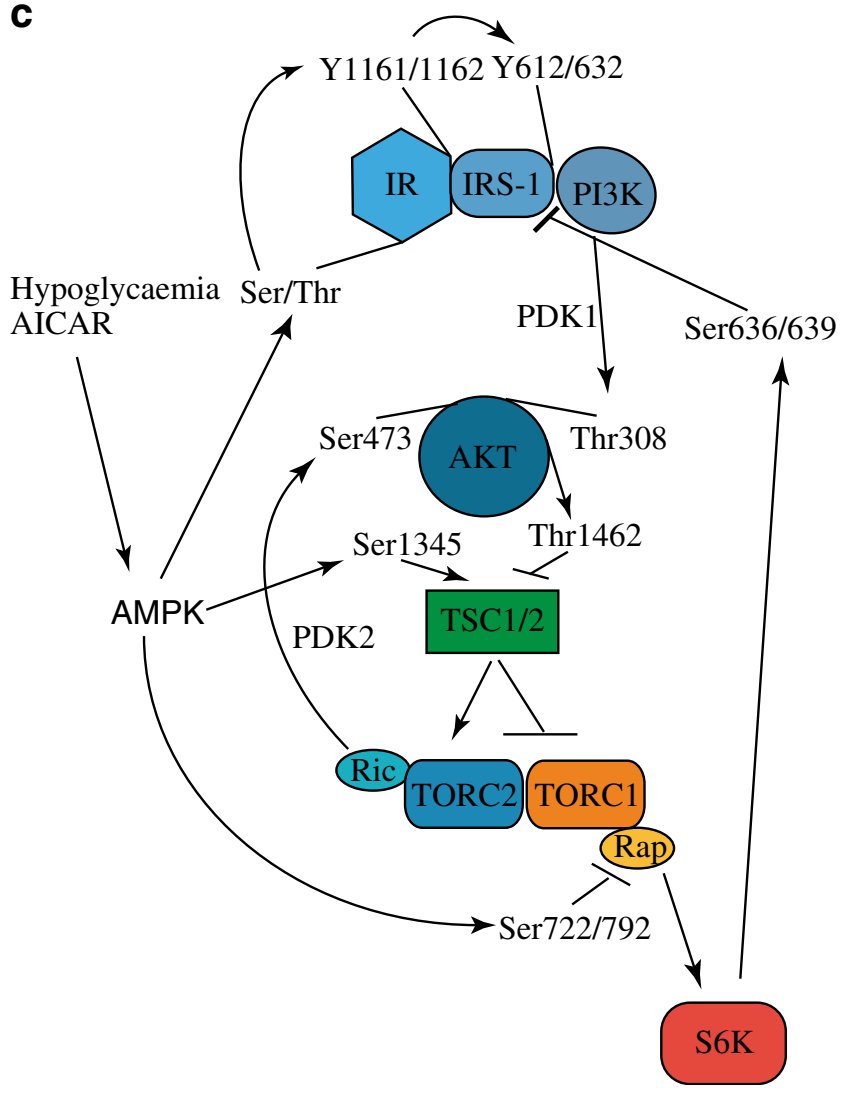

glucose starvation would augment glucose transport in an AMPK- and PI3K-dependent manner. To test for this, we measured DOG uptake during the initial $5 \mathrm{~min}$ of reintroduction in the presence and absence of PI3K and AMPK inhibitors. As shown in Fig. 8b, glucose uptake was increased by more than threefold by glucose-free culture, compared with normal glucose culture. The enhanced glucose uptake was significantly inhibited by infection with dnAMPK or by treatments with the PI3K inhibitors, LY294002 and wortmannin. Combined dnAMPK and LY294002 was no different from either treatment alone, confirming that a component of glucose
4 Fig. 8 Role of activated Akt in ATP homeostasis and glucose uptake. a Cardiac myocytes were cultured as described (Fig. 1a) and incubated with or without glucose for 4,6 or $8 \mathrm{~h}$. Where indicated, cultures were treated with LY294002 (Ly, $20 \mu \mathrm{mol} / \mathrm{l}$ ) or Akt inhibitor X (X, $10 \mu \mathrm{mol} / \mathrm{l})$ for the duration of the experiment. The lysates were collected and ATP concentration measured; $n=5 ; * * * p<0.001$. b Cardiac myocytes were cultured as described (Fig. 1a) and exposed to glucose- and insulin-free conditions for $20 \mathrm{~h}$, followed by glucose uptake quantification. To inhibit AMPK, cultures were pre-infected with 20 plaque forming units (pfu)/cell of adenoviral-dnAMPK for $48 \mathrm{~h}$ before incubations, as described (Fig. 3a). LY294002 (20 $\mu \mathrm{mol} / \mathrm{l})$, wortmannin (Wm, $10 \mu \mathrm{mol} / \mathrm{l})$ or cytochalasin B (Cyto-Cal B, $10 \mathrm{mmol} / \mathrm{l}$ ) were added $30 \mathrm{~min}$ before measurement of glucose uptake; $n=5$; ${ }^{* *} p<0.001$ relative to + glucose condition: ${ }^{\dagger} p<0.005$ relative to control. $\mathbf{c}$ Proposed pathway of glucose starvation/AMPK-mediated sustained activation of Akt. AMPK is activated in muscle cells by multiple physiological and pathophysiological conditions, including glucose deprivation and contraction. We propose that AMPK phosphorylates IR on Ser/Thr residue(s) and promotes allosteric activation of IRK with tyrosine phosphorylation of IR and IRS-1, activation of PI3K and phosphoinositide-dependent kinase 1 (PDK1), and phosphorylation of Akt on Thr308. AMPK also phosphorylates TSC2 on Ser1235 and TORC1-raptor (Rap) on Ser722 and -792, both of which suppress the activation of S6K by Akt. Suppression of S6K blocks the negative feedback termination of insulin signalling and allows sustained activation of Akt by AMPK. TORC2/rictor (Ric) is not affected by AMPK and PDK2 remains active, allowing sustained phosphorylation of Akt-Ser473. AMPK-mediated sustained activation of Akt and suppression of mammalian TORC1 supports retention of the survival functions of Akt, while suppressing the anabolic functions that would be detrimental for cell survival under compromised energy states

transport is common to both inhibitors. To determine whether the PI3K-dependent component of glucose transport involved residual insulin, we also exposed myocytes to saturating levels of an anti-insulin blocking antibody for $1 \mathrm{~h}$ before measuring glucose uptake. This treatment did not significantly reduce glucose uptake, confirming insulin independence (Fig. 8b). Cytochalasin B treatment confirmed a low non-specific background radioactivity associated with the assay.

\section{Discussion}

We have shown that glucose and insulin starvation of cardiac and skeletal myocytes activates AMPK and stimulates the insulin-signalling pathway with five- to tenfold sustained increases in the phosphorylation of Akt and GSK3 $\beta$. During the same time period, the phosphorylation of mammalian TORC1-Ser2448 and S6K-Thr389 decreased. Experiments using selective AMPK inhibitors or AICAR confirmed that AMPK was essential for glucose starvation to activate the insulin signalling pathway. Importantly, the results with different chemical and genetic inhibitors and inducers of AMPK were internally consistent in supporting these conclusions. The effects could not be accounted for by AMPK-mediated phosphorylation of IRS-1 at Ser789 because this was shown to confer negative regulation (Fig. 4). To further investigate the mechanism, we measured the phosphorylation of key tyrosine residues of IRS-1 and IR (Figs 5 and 6). These 
results revealed significantly increased phosphorylation of IRS-1 residues Y612 and Y632, and of IR residue Y1162 by glucose starvation or AICAR. In vitro kinase assays revealed AMPK-dependent IR phosphorylation, which was fully inhibited by compound C. We conclude that AMPK can phosphorylate the human IR. Whereas we have not identified the site of phosphorylation, the human IR contains a number of sequences with homology to the AMPK consensus [37]. We propose that AMPK phosphorylation of the IR causes allosteric activation of IRK independently of insulin. Previous work has shown that the IR is phosphorylated by PKC [42, 43] and cyclic AMP-dependent kinase [44], and that the activation loop of the IR is allosterically modified by ATP binding [45]

Glucose starvation conferred sustained activation of Akt and GSK $3 \beta$, while depressing the phosphorylation of mammalian TORC1 and S6K. These effects can be explained by the dual AMPK-mediated phosphorylation of IR and TSC2. Figure $8 \mathrm{c}$ shows our proposed scheme for regulation of the insulin signalling pathway by glucose starvation. The pathway is initiated by AMPK phosphorylation and allosteric activation of IRK. Activated IRK leads to classical PI3K and Akt activation, but the signal ends there because $\mathrm{S} 6 \mathrm{~K}$ is suppressed due to AMPK-mediated phosphorylation of TSC2 and/or raptor, and repression of mammalian TORC1. S6K normally terminates the insulin signal by phosphorylating IRS-1 at the negative regulatory Ser636 and Ser639 site. The negative feedback regulation does not occur when the pathway is activated by AMPK because IRS-1 activity is protected. In data not shown, we found that when insulin stimulation was superimposed on glucose-starved myocytes, the negative feedback loop was restored and Akt activity returned to its basal unstimulated state by $30 \mathrm{~min}$ after stimulation. This may be due in part to the fact that Akt phosphorylation of TSC overrides that of AMPK, and in part to inhibition of AMPK by Akt [46, 47]. Mammalian TORC2 appears to be fully activated by glucose starvation or AICAR, as evidenced by the sustained phosphorylation of Akt-Ser473 (Fig. 8c).

Our results confirm previous work that the phosphorylation of IRS-1 at Ser789 confers negative regulation and excludes this as a mechanism for activation of the pathway by AMPK $[18,19,21,22]$. We provide direct evidence for negative regulation of IRS-1-Ser789. Our results, moreover, indicate that AMPK activates the pathway despite, rather than because of IRS-1-Ser789. Although positive regulatory IRS-1-Ser/Thr sites have been reported [11, 17, 48, 49], none of these sites contain sequences with significant homology to the AMPK consensus. Consequently, we attribute activation of the insulin signalling pathway by glucose deprivation of cardiac and skeletal myocytes to AMPKmediated phosphorylation of the IR, allosteric activation of IRK and transmission of the signal to IRS-1, PI3K and AktGSK $3 \beta$. Our results further suggest that this improves ATP conservation and augments glucose uptake. Our preliminary observations that this pathway is activated by the same stimuli in skeletal myofilaments suggest that it may translate into similar physiological responses in more complex tissues and whole organs.

In summary, we provide the first evidence that AMPK phosphorylates and activates the IR and promotes activation of the insulin signalling pathway in the absence of insulin or growth factors in cardiac and skeletal myocytes, and in primary skeletal myofilaments. The pathway confers survival signals for cells under energy stress with sustained activation of Akt and GSK $3 \beta$, and suppression of the anabolic pathways regulated by mTOR1 and S6K.

Funding This work was supported by grants HL44578 and HL69812 (to K.A. Webster) from the National Institutes of Health and a grant from the Florida Heart Institute. H. Li and I. Chopra are recipients of American Heart Association, Florida Affiliate predoctoral fellowships. K.A. Webster holds the Walter G. Ross Distinguished Chair in Vascular Biology at the University of Miami Miller School of Medicine.

Duality of interest The authors declare that there is no duality of interest associated with this manuscript.

Contribution statement IC, HL and HW implemented the research, interpreted results, contributed conceptually to manuscript revisions and approved the final manuscript. KAW designed the experiments, interpreted results, wrote the manuscript and approved the final manuscript.

\section{References}

1. Gallagher EJ, Leroith D, Karnieli E (2010) Insulin resistance in obesity as the underlying cause for the metabolic syndrome. Mt Sinai J Med 77:511-523

2. Steinberg GR, Kemp BE (2009) AMPK in health and disease. Physiol Rev 89:1025-1078

3. King MJ, Sale GJ (1990) Dephosphorylation of insulin-receptor autophosphorylation sites by particulate and soluble phosphotyrosylprotein phosphatases. Biochem J 266:251-259

4. Gupte A, Mora S (2006) Activation of the Cbl insulin signaling pathway in cardiac muscle; dysregulation in obesity and diabetes. Biochem Biophys Res Commun 342:751-757

5. Kanzaki M, Mora S, Hwang JB, Saltiel AR, Pessin JE (2004) Atypical protein kinase C (PKCzeta/lambda) is a convergent downstream target of the insulin-stimulated phosphatidylinositol 3-kinase and TC10 signaling pathways. J Cell Biol 164:279-290

6. Vasudevan KM, Garraway LA (2010) AKT signaling in physiology and disease. Curr Top Microbiol Immunol 347:105-133

7. Abel ED (2004) Glucose transport in the heart. Front Biosci 9:201-215

8. Matsui T, Rosenzweig A (2005) Convergent signal transduction pathways controlling cardiomyocyte survival and function: the role of PI 3-kinase and Akt. J Mol Cell Cardiol 38:63-71

9. Shiraishi I, Melendez J, Ahn Y et al (2004) Nuclear targeting of Akt enhances kinase activity and survival of cardiomyocytes. Circ Res 94:884-891

10. Juhaszova M, Zorov DB, Yaniv Y, Nuss HB, Wang S, Sollott SJ (2009) Role of glycogen synthase kinase-3beta in cardioprotection. Circ Res 104:1240-1252 
11. Gual P, Le Marchand-Brustel Y, Tanti JF (2005) Positive and negative regulation of insulin signaling through IRS-1 phosphorylation. Biochimie 87:99-109

12. Weigert C, Kron M, Kalbacher $\mathrm{H}$ et al (2008) Interplay and effects of temporal changes in the phosphorylation state of serine-302, -307 , and -318 of insulin receptor substrate-1 on insulin action in skeletal muscle cells. Mol Endocrinol 22:2729-2740

13. Bouzakri K, Koistinen HA, Zierath JR (2005) Molecular mechanisms of skeletal muscle insulin resistance in type 2 diabetes. Curr Diabetes Rev 1:167-174

14. Bossenmaier B, Strack V, Stoyanov B et al (2000) Serine residues $1177 / 78 / 82$ of the insulin receptor are required for substrate phosphorylation but not autophosphorylation. Diabetes 49:889-895

15. Um SH, D'Alessio D, Thomas G (2006) Nutrient overload, insulin resistance, and ribosomal protein S6 kinase 1, S6K1. Cell Metab 3:393-402

16. Um SH, Frigerio F, Watanabe M et al (2004) Absence of S6K1 protects against age- and diet-induced obesity while enhancing insulin sensitivity. Nature 431:200-205

17. Zick Y (2005) Ser/Thr phosphorylation of IRS proteins: a molecular basis for insulin resistance. Sci STKE 2005:pe4

18. Qiao LY, Zhande R, Jetton TL, Zhou G, Sun XJ (2002) In vivo phosphorylation of insulin receptor substrate 1 at serine 789 by a novel serine kinase in insulin-resistant rodents. J Biol Chem 277:26530-26539

19. Horike N, Takemori H, Katoh Y et al (2003) Adipose-specific expression, phosphorylation of Ser794 in insulin receptor substrate-1, and activation in diabetic animals of salt-inducible kinase-2. J Biol Chem 278:18440-18447

20. Jakobsen SN, Hardie DG, Morrice N, Tornqvist HE (2001) 5'AMP-activated protein kinase phosphorylates IRS-1 on Ser-789 in mouse $\mathrm{C} 2 \mathrm{C} 12$ myotubes in response to 5-aminoimidazole-4-carboxamide riboside. J Biol Chem 276:46912-46916

21. Zakikhani M, Blouin MJ, Piura E, Pollak MN (2010) Metformin and rapamycin have distinct effects on the AKT pathway and proliferation in breast cancer cells. Breast Cancer Res Treat 123:271-279

22. Ning J, Clemmons DR (2010) AMP-activated protein kinase inhibits IGF-I signaling and protein synthesis in vascular smooth muscle cells via stimulation of insulin receptor substrate 1 S794 and tuberous sclerosis 2 S1345 phosphorylation. Mol Endocrinol 24:1218-1229

23. Hardie DG, Hawley SA, Scott JW (2006) AMP-activated protein kinase - development of the energy sensor concept. J Physiol 574:7-15

24. Arad M, Seidman CE, Seidman JG (2007) AMP-activated protein kinase in the heart: role during health and disease. Circ Res 100:474-488

25. Zhang BB, Zhou G, Li C (2009) AMPK: an emerging drug target for diabetes and the metabolic syndrome. Cell Metab 9:407-416

26. Walker PS, Ramlal T, Sarabia V et al (1990) Glucose transport activity in L6 muscle cells is regulated by the co-ordinate control of subcellular glucose transporter distribution, biosynthesis, and mRNA transcription. J Biol Chem 265:1516-1523

27. Sasson S, Kaiser N, Dan-Goor M et al (1997) Substrate autoregulation of glucose transport: hexose 6-phosphate mediates the cellular distribution of glucose transporters. Diabetologia 40:30-39

28. Itani SI, Saha AK, Kurowski TG, Coffin HR, Tornheim K, Ruderman NB (2003) Glucose autoregulates its uptake in skeletal muscle: involvement of AMP-activated protein kinase. Diabetes 52:1635-1640

29. Towler MC, Hardie DG (2007) AMP-activated protein kinase in metabolic control and insulin signaling. Circ Res 100:328-341

30. Kubasiak LA, Hernandez OM, Bishopric NH, Webster KA (2002) Hypoxia and acidosis activate cardiac myocyte death through the Bcl-2 family protein BNIP3. Proc Natl Acad Sci U S A 99:12825-12830

31. Takikita S, Myerowitz R, Zaal K, Raben N, Plotz PH (2009) Murine muscle cell models for Pompe disease and their use in studying therapeutic approaches. Mol Genet Metab 96:208-217
32. Luo M, Reyna S, Wang L et al (2005) Identification of insulin receptor substrate 1 serine/threonine phosphorylation sites using mass spectrometry analysis: regulatory role of serine 1223. Endocrinology 146:4410-4416

33. Chaudary N, Naydenova Z, Shuralyova I, Coe IR (2004) Hypoxia regulates the adenosine transporter, mENT1, in the murine cardiomyocyte cell line, HL-1. Cardiovasc Res 61:780-788

34. Goodyear LJ, Giorgino F, Sherman LA, Carey J, Smith RJ, Dohm GL (1995) Insulin receptor phosphorylation, insulin receptor substrate-1 phosphorylation, and phosphatidylinositol 3-kinase activity are decreased in intact skeletal muscle strips from obese subjects. J Clin Invest 95:2195-2204

35. McManus EJ, Alessi DR (2002) TSC1-TSC2: a complex tale of PKB-mediated S6K regulation. Nat Cell Biol 4:E214-E216

36. Harrington LS, Findlay GM, Lamb RF (2005) Restraining PI3K: mTOR signalling goes back to the membrane. Trends Biochem Sci $30: 35-42$

37. Gwinn DM, Shackelford DB, Egan DF et al (2008) AMPK phosphorylation of raptor mediates a metabolic checkpoint. Mol Cell 30:214-226

38. Sarbassov DD, Guertin DA, Ali SM, Sabatini DM (2005) Phosphorylation and regulation of Akt/PKB by the rictor-mTOR complex. Science 307:1098-1101

39. Esposito DL, Li Y, Cama A, Quon MJ (2001) Tyr(612) and Tyr (632) in human insulin receptor substrate-1 are important for full activation of insulin-stimulated phosphatidylinositol 3-kinase activity and translocation of GLUT4 in adipose cells. Endocrinology $142: 2833-2840$

40. Desbois C, Capeau J, Hainault I et al (1992) Differential role of insulin receptor autophosphorylation sites 1162 and 1163 in the long-term insulin stimulation of glucose transport, glycogenesis, and protein synthesis. J Biol Chem 267:13488-13497

41. Hahn-Windgassen A, Nogueira V, Chen CC, Skeen JE, Sonenberg N, Hay N (2005) Akt activates the mammalian target of rapamycin by regulating cellular ATP level and AMPK activity. J Biol Chem 280:32081-32089

42. Bollag GE, Roth RA, Beaudoin J, Mochly-Rosen D, Koshland DE Jr (1986) Protein kinase C directly phosphorylates the insulin receptor in vitro and reduces its protein-tyrosine kinase activity. Proc Natl Acad Sci U S A 83:5822-5824

43. Pillay TS, Xiao S, Keranen L, Olefsky JM (2004) Regulation of the insulin receptor by protein kinase $\mathrm{C}$ isoenzymes: preferential interaction with beta isoenzymes and interaction with the catalytic domain of betaII. Cell Signal 16:97-104

44. Maddux BA, Goldfine ID (1991) Evidence that insulin plus ATP may induce a conformational change in the beta subunit of the insulin receptor without inducing receptor autophosphorylation. J Biol Chem 266:6731-6736

45. Roth RA, Beaudoin J (1987) Phosphorylation of purified insulin receptor by cAMP kinase. Diabetes 36:123-126

46. Horman S, Vertommen D, Heath R (2006) Insulin antagonizes ischemia-induced Thr172 phosphorylation of AMP-activated protein kinase alpha-subunits in heart via hierarchical phosphorylation of Ser485/491. J Biol Chem 281:5335-5340

47. Manning BD, Tee AR, Logsdon MN, Blenis J, Cantley LC (2002) Identification of the tuberous sclerosis complex-2 tumor suppressor gene product tuberin as a target of the phosphoinositide 3-kinase/akt pathway. Mol Cell 10:151-162

48. Greene MW, Sakaue H, Wang L, Alessi DR, Roth RA (2003) Modulation of insulin-stimulated degradation of human insulin receptor substrate-1 by serine 312 phosphorylation. J Biol Chem 278:8199-8211

49. Giraud J, Leshan R, Lee YH, White MF (2004) Nutrientdependent and insulin-stimulated phosphorylation of insulin receptor substrate-1 on serine 302 correlates with increased insulin signaling. J Biol Chem 279:3447-3454 